\title{
The Zeros of Polar Derivative of Polynomials with Respect to Real Number
}

\author{
K. Sravani ${ }^{1}$, K. Anoosha ${ }^{2}$, G. L. Reddy $^{3}$ \\ ${ }^{1,2,3}$ School of Mathematics and Statistics, University of Hyderabad - 500046, India
}

\begin{abstract}
In this paper we obtain the size of the disc in which the zeros of polar derivatives of polynomial of degree $n$ with real coefficients with respect to a real a lie.
\end{abstract}

Keywords: zeros, polar derivatives, polynomials, real $\alpha$.

\section{Introduction}

To estimate the zeros of a polynomial is a long standing problem. It is an interesting area of research for many engineers as well as mathematicians and many results on the topic are available in the literature.

If $\mathrm{P}(\mathrm{z})=\sum_{i=0}^{n} a_{\mathrm{i}} \mathrm{z}$, be a polynomial of degree $\mathrm{n}$ then Polar Derivative of the polynomial $\mathrm{P}(\mathrm{z})$ with respect to $\alpha$, where $\alpha$ can be real or complex number, is defined as

$D_{\alpha} P(z)=n P(z)+(\alpha-z) P^{\prime}(z)$

It is a polynomial of degree up to $n-1$. The polynomial $\mathrm{D}_{\alpha} \mathrm{P}(\mathrm{z})$ generalizes the ordinary derivative, in the sense that $\lim _{\alpha \rightarrow \infty} \mathrm{D}_{\alpha} \mathrm{P}(\mathrm{z}) / \alpha=\mathrm{P}^{\prime}(\mathrm{z})$.

The well-known results on Eneström-Kakeya theorem (see $[1,2])$ in theory of distribution of zeros of polynomials are the following.

Theorem (1): Let $\mathrm{P}(\mathrm{z})=\sum_{i=0}^{n} a_{\mathrm{i}} \mathrm{z}^{\mathrm{i}}$ be a polynomial of degree n such that

$0<\mathrm{a}_{0} \leq \mathrm{a}_{1} \leq \ldots \leq \mathrm{a}_{\mathrm{n}}$

Then all the zeros of $P(z)$ lie in $|z| \leq 1$.

A. Joyal , G. Labelle and Q.I.Rahman [3] obtained the following generalization, by considering the coefficients to be real instead of being only positive.

Theorem (2): Let $\mathrm{P}(\mathrm{z})=\sum_{i=0}^{n} a_{\mathrm{i}} \mathrm{z}^{\mathrm{i}}$ be a polynomial of degree n such that

\section{$\mathrm{a}_{0} \leq \mathrm{a}_{1} \leq \ldots \leq \mathrm{a}_{\mathrm{n}}$.}

Then all the zeros of $P(z)$ lie in $|z| \leq\left|a_{n}\right|^{-1}\left\{a_{n}-a_{0}+\left|a_{0}\right|\right\}$.

This paper we prove the following results.

Aziz and Zargar [1] relaxed the hypothesis of Enestr $\square$ om-

Kakeya theorem in

a different way and proved the following results.

Theorem (3): Let $\mathrm{P}(\mathrm{z})=\sum_{i=0}^{n} a_{\mathrm{i}} \mathrm{z}^{\mathrm{i}}$ be a polynomial of degree $\mathrm{n}$ such that $\mathrm{k} \geq 1$,

$0<\mathrm{a}_{0} \leq \mathrm{a}_{1} \leq \ldots \leq \mathrm{a}_{\mathrm{n}-1} \leq \mathrm{ka} \mathrm{a}_{\mathrm{n}}$.

Then all the zeros of $\mathrm{P}(\mathrm{z})$ lie in $|\mathrm{z}+\mathrm{k}-1| \leq \mathrm{k}$.

Theorem (4): Let $\mathrm{P}(\mathrm{z})=\sum_{i=0}^{n} a_{\mathrm{i}} \mathrm{z}^{\mathrm{i}}$ be a polynomial of degree $\mathrm{n}$ such that $\mathrm{k} \geq 1$,

$\mathrm{a}_{0} \leq \mathrm{a}_{1} \leq \ldots \leq \mathrm{a}_{\mathrm{n}-1} \leq \mathrm{ka} \mathrm{a}_{\mathrm{n}}$

Then all the zeros of $\mathrm{P}(\mathrm{z})$ lie in $|\mathrm{z}+\mathrm{k}-1| \leq\left|\mathrm{a}_{\mathrm{n}}\right|^{-1}\left\{\mathrm{ka}_{\mathrm{n}}-\mathrm{a}_{0}+\left|\mathrm{a}_{0}\right|\right\}$.

\section{Zeros of Polar Derivative of Polynomial P(z)}

This section is concerned with the location of the zeros of the polar derivative of a polynomial with real coeffcients with respect to a real number.

Theorem (5): Let $\mathrm{P}(\mathrm{z})=\sum_{i=0}^{n} a_{\mathrm{i}} \mathrm{z}^{\mathrm{i}}$ be a polynomial of degree n such that $\mathrm{k} \geq 1$,

$\mathrm{a}_{0} \leq \mathrm{a}_{1} \leq \ldots \leq \mathrm{a}_{\mathrm{n}-1} \leq \mathrm{ka} \mathrm{a}_{\mathrm{m}} \geq \mathrm{a}_{\mathrm{m}+1} \geq \ldots \mathrm{a}_{\mathrm{n}-1} \geq \mathrm{a}_{\mathrm{n}}$

$(n-i) a_{i} \leq a_{i+1}, i=0,1,2, \ldots . m-1$

$\mathrm{ja}_{\mathrm{j}} \leq(\mathrm{j}-1) \mathrm{a}_{\mathrm{j}-1, \mathrm{j}} \mathrm{j}=\mathrm{m}+1, \ldots \mathrm{n}$.

Then for a real $\alpha$ the polar derivatives of $\mathrm{P}(\mathrm{z})$ with respect to $\alpha$ has at most $n-1$ zeros and they lie in

$|\mathrm{z}| \leq\left|\mathrm{a}_{\mathrm{n}-1}+\alpha n \mathrm{a}_{\mathrm{n}}\right|^{-1}\left\{-\mathrm{a}_{\mathrm{n}-1}-\alpha n \mathrm{a}_{\mathrm{n}}+\mathrm{k}(\mathrm{n}-\mathrm{m}) \mathrm{a}_{\mathrm{m}}+\alpha(\mathrm{m}+1) \mathrm{a}_{\mathrm{m}+1}+(\mathrm{n}-\right.$ $2 \mathrm{~m})(\mathrm{k}-1)\left|\mathrm{a}_{\mathrm{m}}\right|+$

$\left|(n-m) a_{m}+\quad \alpha(m+1) a_{m+1}-(n-m+1) a_{m-1}-\alpha m a_{m}\right|+(n-m+1) a_{m-}$ $\left.{ }_{1}+\mathrm{k}_{\mathrm{m}} \mathrm{ma}_{\mathrm{m}}-\mathrm{na}_{0}-\alpha \mathrm{a}_{1}+\left|\mathrm{na}_{0}+\alpha \mathrm{a}_{1}\right|\right\}$.

Proof: Let $\mathrm{P}(\mathrm{z})=\mathrm{a}_{0}+\mathrm{a}_{1} \mathrm{z}+\mathrm{a}_{2} \mathrm{z}^{2}+\ldots \ldots+\mathrm{a}_{\mathrm{n}} \mathrm{z}^{\mathrm{n}}$ be $\mathrm{a}$ polynomial of degree $n$.

Then the polar derivative of $\mathrm{P}(\mathrm{z})$ is given by $\mathrm{D}_{\alpha} \mathrm{P}(\mathrm{z})=\mathrm{nP}(\mathrm{z})$ $+(\alpha-z) P^{\prime}(z)$

Then

$\mathrm{D}_{\alpha} \mathrm{P}(\mathrm{z})==\left[\mathrm{na}_{0}+\alpha \mathrm{a}_{1}\right]+\left[(\mathrm{n}-1) \mathrm{a}_{1}+2 \alpha \mathrm{a}_{2}\right] \mathrm{z}+\left[(\mathrm{n}-2) \mathrm{a}_{2}+\right.$ $\left.3 \alpha \mathrm{a}_{3}\right] \mathrm{z}^{2}+\ldots$

$+\left[(n-m+1) a_{m-1}+\alpha m a_{m}\right] z^{m-1}+\left[(n-m) a_{m}+\alpha(m+1) a_{m+1}\right] z^{m}+$ $\left[(\mathrm{n}-\mathrm{m}-1) \mathrm{a}_{\mathrm{m}+1}+\alpha(\mathrm{m}+2) \mathrm{a}_{\mathrm{m}+2}\right] \mathrm{z}^{\mathrm{m}+1}+$

$+\left[2 a_{n-2}+\alpha(n-1) a_{n-1}\right] z^{n-2}+\left[a_{n-1}+\alpha n a_{n}\right] z^{n-1}$.

Now consider the polynomial $\mathrm{Q}(\mathrm{z})=(1-\mathrm{z}) \mathrm{D}_{\alpha} \mathrm{P}(\mathrm{z})$ so that $\mathrm{Q}(\mathrm{z})=-\left[\mathrm{a}_{\mathrm{n}-1}+\alpha n a_{\mathrm{n}}\right] \mathrm{z}^{\mathrm{n}}+\left[\mathrm{a}_{\mathrm{n}-1}+\alpha n \mathrm{a}_{\mathrm{n}}-2 \mathrm{a}_{\mathrm{n}-2}-\alpha(\mathrm{n}-1) \mathrm{a}_{\mathrm{n}-1}\right] \mathrm{z}^{\mathrm{n}-1}+$

$+\left[(n-m-1) a_{m+1}+\alpha(m+2) a_{m+2}-(n-m) a_{m}-\alpha(m+1) a_{m+1}\right] z^{m+1}$

$+\left[(n-m) a_{m}+\alpha(m+1) a_{m+1}-(n-m+1) a_{m-1}-\alpha m a_{m}\right] z^{m}$

$+\left[(n-m+1) a_{m-1}+\alpha m a_{m}-(n-m+2) a_{m-2}-\alpha(m-1) a_{m-1}\right] z^{m-1}+$

$+\left[(n-2) a_{2}+3 \alpha a_{3}-(n-1) a_{1}-2 \alpha a_{2}\right] z^{2}+\left[(n-1) a_{1}+2 \alpha a_{2}-n a_{0}\right.$ $\left.-\alpha \mathrm{a}_{1}\right] \mathrm{z}$

$+\left[\mathrm{na}_{0}+\alpha \mathrm{a}_{1}\right]$.

Now if $|z|>1$ then $|z|^{-(n-i)}<1$ for $i=1,2,3, \ldots . . n-1$

Further

$|\mathrm{Q}(\mathrm{z})| \geq\left|\mathrm{a}_{\mathrm{n}-1}+\alpha n \mathrm{a}_{\mathrm{n}}\right||\mathrm{z}|^{\mathrm{n}}-\left\{\left|\mathrm{a}_{\mathrm{n}-1}+\alpha n \mathrm{a}_{\mathrm{n}}-2 \mathrm{a}_{\mathrm{n}-2}-\alpha(\mathrm{n}-1) \mathrm{a}_{\mathrm{n}-1}\right||\mathrm{z}|^{\mathrm{n}-1}\right.$ $+\ldots+\left|(n-m-1) a_{m+1}+\alpha(m+2) a_{m+2}-(n-m) a_{m}-\alpha(m+1) a_{m+1}\right|$ $|z|^{\mathrm{m}+1}$ 


\section{International Journal of Science and Research (IJSR) \\ ISSN (Online): 2319-7064}

Index Copernicus Value (2013): 6.14 | Impact Factor (2014): 5.611

$+\left|(n-m) a_{m}+\alpha(m+1) a_{m+1}-(n-m+1) a_{m-1}-\alpha m a_{m}\right||z|^{m}$

$+\left|(n-m+1) a_{m-1}+\alpha m a_{m}-(n-m+2) a_{m-2}-\alpha(m-1) a_{m-1}\right||z|^{m-1}$

$+\left|(n-2) a_{2}+3 \alpha a_{3}-(n-1) a_{1}-2 \alpha a_{2}\right||z|^{2}+\mid(n-1) a_{1}+2 \alpha a_{2}-n a_{0}-$

$\alpha \mathrm{a}_{1}|\mathrm{z}|$

$\left.+\left|\mathrm{na}_{0}+\alpha \mathrm{a}_{1}\right|\right\}$.

$\geq\left|a_{n-1}+\alpha n a_{n}\right||z|^{n-1}\left[|z|-\left|a_{n-1}+\alpha n a_{n}\right|^{-1}\left\{\mid a_{n-1}+\alpha n a_{n}-2 a_{n-2}\right.\right.$ $-\alpha(n-1) a_{n-1}|+| 2 a_{n-2}+\alpha(n-1) a_{n-1}-3 a_{n-3}-\left.\alpha(n-2) a_{n-2}|| z\right|^{-1}+\ldots$ $+\left|(n-m-1) a_{m+1}+\alpha(m+2) a_{m+2}-(n-m) a_{m}-\alpha(m+1) a_{m+1}\right||z|^{-(n-m-}$ 2)

$+\left|(n-m) a_{m}+\alpha(m+1) a_{m+1}-(n-m+1) a_{m-1}-\alpha m a_{m} \| z\right|^{-(n-m-1)}$

$+\left|(n-m+1) a_{m-1}+\alpha m a_{m}-(n-m+2) a_{m-2}-\alpha(m-1) a_{m-1}\right||z|^{-(n-m)}+$

$\cdots$

$+\left|(n-2) a_{2}+3 \alpha a_{3}-(n-1) a_{1}-2 \alpha a_{2}\right||z|^{-(n-3)}+\mid(n-1) a_{1}+2 \alpha a_{2}$

$\left.\left.-n a_{0}-\left.\alpha a_{1}|| z\right|^{-(n-2)}+\left|n a_{0}+\alpha a_{1}\right||z|^{-(n-1)}\right\}\right]$.

$\geq\left|a_{n-1}+\alpha n a_{n}\right||z|^{n-1}\left[|z|-\left|a_{n-1}+\alpha n a_{n}\right|^{-1}\left\{\mid a_{n-1}+\alpha n a_{n}-2 a_{n-2}\right.\right.$ $-\alpha(n-1) a_{n-1}|+| 2 a_{n-2}+\alpha(n-1) a_{n-1}-3 a_{n-3}-\alpha(n-2) a_{n-2} \mid+\ldots$ $+\left|(n-m-1) a_{m+1}+\alpha(m+2) a_{m+2}-(n-m) a_{m}-\alpha(m+1) a_{m+1}\right|$

$+\left|(n-m) a_{m}+\alpha(m+1) a_{m+1}-(n-m+1) a_{m-1}-\alpha m a_{m}\right|$

$+\left|(n-m+1) a_{m-1}+\alpha m a_{m}-(n-m+2) a_{m-2}-\alpha(m-1) a_{m-1}\right|+\ldots$

$+\left|(n-2) a_{2}+3 \alpha a_{3}-(n-1) a_{1}-2 \alpha a_{2}\right|+\mid(n-1) a_{1}+2 \alpha a_{2}-n a_{0}-$ $\left.\left.\alpha \mathrm{a}_{1}|+| \mathrm{na}_{0}+\alpha \mathrm{a}_{1} \mid\right\}\right]$.

$\geq\left|a_{n-1}+\alpha n a_{n}\right||z|^{n-1}\left[|z|-\left|a_{n-1}+\alpha n a_{n}\right|^{-1}\left\{\mid a_{n-1}+\alpha n a_{n}-2 a_{n-2}\right.\right.$

$-\alpha(n-1) a_{n-1}|+| 2 a_{n-2}+\alpha(n-1) a_{n-1}-3 a_{n-3}-\alpha(n-2) a_{n-2} \mid+\ldots$

$+\mid(n-m-1) a_{m+1}+\alpha(m+2) a_{m+2}-k(n-m) a_{m}-\alpha(m+1) a_{m+1}$

$+k(n-m) a_{m}-(n-m) a_{m}|+|(n-m) a_{m}+\alpha(m+1) a_{m+1}-(n-m+1) a_{m-1}-$ $\alpha \mathrm{ma}_{\mathrm{m}}$

$+\mid(n-m+1) a_{m-1}+\alpha k m a_{m}-\alpha k_{m a}+\alpha m a_{m}-(n-m+2) a_{m-2}-\alpha(m-$ 1) $\mathrm{a}_{\mathrm{m}-1} \mid+\ldots$

$+\left|(n-2) a_{2}+3 \alpha a_{3}-(n-1) a_{1}-2 \alpha a_{2}\right|+\mid(n-1) a_{1}+2 \alpha a_{2}-n a_{0}-$ $\alpha \mathrm{a}_{1} \mid$

$\left.\left.+\left|\mathrm{na}_{0}+\alpha \mathrm{a}_{1}\right|\right\}\right]$.

$\geq\left|a_{n-1}+\alpha n a_{n}\right||z|^{n-1}\left(|z|-\left|a_{n-1}+\alpha n a_{n}\right|^{-1}\left\{\left[2 a_{n-2}+\alpha(n-1) a_{n-1} \quad-a_{n-1}{ }^{-}\right.\right.\right.$ $\left.\alpha n a_{n}\right]+\left[3 a_{n-3}+\alpha(n-2) a_{n-2}\right.$

$\left.-2 a_{n-2}-\alpha(n-1) a_{n-1}\right]+\ldots \ldots++\left[k(n-m) a_{m}+\alpha(m+1) a_{m+1}-(n-m-\right.$

1) $\mathrm{a}_{\mathrm{m}+1}$

$\left.-\alpha(m+2) a_{m+2}\right]+(k-1)(n-m)\left|a_{m}\right|+\mid(n-m) a_{m}+\alpha(m+1) a_{m+1}$

$-(n-m+1) a_{m-1}-\alpha m a_{m} \mid+\left[(n-m+1) a_{m-1}+\alpha k m a_{m}-(n-m+2) a_{m-2}\right.$

$\left.-\alpha(m-1) a_{m-1}+(1-k)\left|a_{m}\right|\right]+\ldots .+\left[(n-2) a_{2}+3 \alpha a_{3}-(n-1) a_{1}-2 \alpha a_{2}\right]$

$\left.\left.+\left[(\mathrm{n}-1) \mathrm{a}_{1}+2 \alpha \mathrm{a}_{2}-\mathrm{na}_{0}-\alpha \mathrm{a}_{1}\right]+\left|n \mathrm{n}_{0}+\alpha \mathrm{a}_{1}\right|\right\}\right)$

$\geq\left|a_{n-1}+\quad \alpha n a_{n}\right||z|^{n-1}\left(|z|-\left|\quad a_{n-1}+\alpha n a_{n}\right|^{-1}\left(-a_{n-1}-\alpha n a_{n}+k(n-m) a_{m}+\right.\right.$ $\alpha(\mathrm{m}+1) \mathrm{a}_{\mathrm{m}+1}+(\mathrm{n}-2 \mathrm{~m})(\mathrm{k}-1)\left|\mathrm{a}_{\mathrm{m}}\right|$

$+\left|(n-m) a_{m}+\alpha(m+1) a_{m+1}-(n-m+1) a_{m-1}-\alpha m a_{m}\right|$

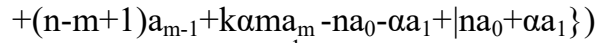

$>0$ if $|\mathrm{z}|>\left|\mathrm{a}_{\mathrm{n}-1}+\alpha n \mathrm{a}_{\mathrm{n}}\right|^{-1}\left(-\mathrm{a}_{\mathrm{n}-1}-\alpha n \mathrm{a}_{\mathrm{n}}+\mathrm{k}(\mathrm{n}-\mathrm{m}) \mathrm{a}_{\mathrm{m}}+\alpha(\mathrm{m}+1) \mathrm{a}_{\mathrm{m}+1}+(\mathrm{n}-\right.$

$2 \mathrm{~m})(\mathrm{k}-1)\left|\mathrm{a}_{\mathrm{m}}\right|$

$+\left|(n-m) a_{m}+\alpha(m+1) a_{m+1}-(n-m+1) a_{m-1}-\alpha m a_{m}\right|$

$\left.+(n-m+1) a_{m-1}+k \alpha m a_{m}-n a_{0}-\alpha a_{1}+\left|n a_{0}+\alpha a_{1}\right|\right)$

This shows that if $|z|>\left|a_{n-1}+\alpha n a_{n}\right|^{-1}\left(-a_{n-1}-\alpha n a_{n}+k(n-m) a_{m}+\right.$ $\alpha(\mathrm{m}+1) \mathrm{a}_{\mathrm{m}+1}+(\mathrm{n}-2 \mathrm{~m})(\mathrm{k}-1)\left|\mathrm{a}_{\mathrm{m}}\right|+\mid(\mathrm{n}-\mathrm{m}) \mathrm{a}_{\mathrm{m}}+\alpha(\mathrm{m}+1) \mathrm{a}_{\mathrm{m}+1}$

$-(n-m+1) a_{m-1}-\alpha m a_{m} \mid+(n-m+1) a_{m-1}+k \alpha m a_{m}$

$-\mathrm{na}_{0^{-}}$

$\left.\alpha \mathrm{a}_{1}+\left|n \mathrm{n}_{0}+\alpha \mathrm{a}_{1}\right|\right)$, then $\mathrm{Q}(\mathrm{z})>0$.

Hence all the zeros of $Q(z)$ with $|z|>1$ lie in

$|z| \leq\left|a_{n-1}+\alpha n a_{n}\right|^{-1}\left(-a_{n-1}-\alpha n a_{n}+k(n-m) a_{m}+\alpha(m+1) a_{m+1} \quad+(n-\right.$ $2 \mathrm{~m})(\mathrm{k}-1)\left|\mathrm{a}_{\mathrm{m}}\right|+\mid(\mathrm{n}-\mathrm{m}) \mathrm{a}_{\mathrm{m}}+\alpha(\mathrm{m}+1) \mathrm{a}_{\mathrm{m}+1}$

$-(n-m+1) a_{m-1}-\alpha m a_{m} \mid+(n-m+1) a_{m-1}+k \alpha m a_{m}$

$\left.\alpha \mathrm{a}_{1}+\left|\mathrm{na}_{0}+\alpha \mathrm{a}_{1}\right|\right)$

But those zeros of $\mathrm{Q}(\mathrm{z})$ whose modulus is less than or equal to 1 ,already satisfy the above inequality, since all the zeros of $\mathrm{D}_{\alpha} \mathrm{P}(\mathrm{z})$ are also the zeros of $\mathrm{Q}(\mathrm{z})$ as they lie in the circle defined by the above inequality.This completes the proof of the theorem.

Corollary 6 : Let $\mathrm{P}(\mathrm{z})=\sum_{i=0}^{n} a_{\mathrm{i}} \mathrm{z}^{\mathrm{i}}$ be a polynomial of degree $\mathrm{n}$ such that $\mathrm{k} \geq 1$,

$a_{0} \leq a_{1} \leq \ldots . \leq k a_{m} \geq a_{m+1} \geq \ldots a_{n-1} \geq a_{n}$

$(n-i) a_{i} \leq a_{i+1}, i=0,1,2, \ldots . m-1$

$\mathrm{ja}_{\mathrm{j}} \leq(\mathrm{j}-1) \mathrm{a}_{\mathrm{j}-1, \mathrm{j}} \mathrm{j}=\mathrm{m}+1, \ldots \mathrm{n}$.

then for a real $\alpha \neq-a_{n-1} / \operatorname{na}_{n}$ the polar derivative of $\mathrm{P}(\mathrm{z})$ with respect to $\alpha$ has exactly $n-1$ zeros and they lie in

$|z| \leq\left|a_{n-1}+\alpha n a_{n}\right|^{-1}\left(-a_{n-1}-\alpha n a_{n}+k(n-m) a_{m}+\alpha(m+1) a_{m+1} \quad+(n-\right.$

$2 \mathrm{~m})(\mathrm{k}-1)\left|\mathrm{a}_{\mathrm{m}}\right|+\mid(\mathrm{n}-\mathrm{m}) \mathrm{a}_{\mathrm{m}}+\alpha(\mathrm{m}+1) \mathrm{a}_{\mathrm{m}+1}$

$-(n-m+1) a_{m-1}-\alpha m a_{m} \mid+(n-m+1) a_{m-1}+k \alpha m a_{m} \quad-n a_{0^{-}}$ $\left.\alpha \mathrm{a}_{1}+\left|\mathrm{na}_{0}+\alpha \mathrm{a}_{1}\right|\right)$

Indeed, if real $\alpha \neq-a_{n-1} / a_{n}$ then $D_{\alpha} P(z)$ is of $n-1$ degree so it has n-1.

Corollary 7 : Let $\mathrm{P}(\mathrm{z})=\sum_{i=0}^{n} a_{\mathrm{i}} \mathrm{z}^{\mathrm{i}}$ be a polynomial of degree $\mathrm{n}$ such that $\mathrm{k} \geq 1$,

$\mathrm{a}_{0} \leq \mathrm{a}_{1} \leq \ldots \leq \mathrm{ka}_{\mathrm{m}} \geq \mathrm{a}_{\mathrm{m}+1} \geq \ldots \mathrm{a}_{\mathrm{n}-1} \geq \mathrm{a}_{\mathrm{n}}$

$(n-i) a_{i} \leq a_{i+1}, i=0,1,2, \ldots . m-1$

$j a_{j} \geq(j-1) a_{j-1}, j=m+1, \ldots n$.

then for a real $\alpha$ the polar derivatives of $\mathrm{P}(\mathrm{z})$ with respect to $\alpha$ has atmost $n-1$ zeros and they lie in $|\mathrm{z}| \leq\left|\mathrm{a}_{\mathrm{n}-1}+\alpha n \mathrm{a}_{\mathrm{n}}\right|^{-1}\left(-\mathrm{a}_{\mathrm{n}-1}-\alpha \mathrm{na}_{\mathrm{n}}+(\mathrm{n}-\mathrm{m}) \mathrm{a}_{\mathrm{m}}+\alpha(\mathrm{m}+1) \mathrm{a}_{\mathrm{m}+1}+\right.$

$\left|(n-m) a_{m}+\alpha(m+1) a_{m+1}-(n-m+1) a_{m-1}-\alpha m a_{m}\right|$

$\left.+(n-m+1) a_{m-1}+\alpha m a_{m}-n a_{0}-\alpha a_{1}+\left|n a_{0}+\alpha a_{1}\right|\right)$

The above corollary is obtained by taking $\mathrm{k}=1$.

Theorem 8: Let $\mathrm{P}(\mathrm{z})=\sum_{i=0}^{n} a_{\mathrm{i}} \mathrm{z}^{\mathrm{i}}$ be a polynomial of degree $\mathrm{n}$ such that $\mathrm{k} \geq 1$,

$\mathrm{a}_{0} \leq \mathrm{a}_{1} \leq \ldots \leq \mathrm{ka}_{\mathrm{m}} \geq \mathrm{a}_{\mathrm{m}+1} \geq \ldots \mathrm{a}_{\mathrm{p}-1} \geq \mathrm{a}_{\mathrm{p}}$

$(n-i) a_{i} \leq a_{i+1}, i=0,1,2, \ldots . m-1$

$\mathrm{ja}_{\mathrm{j}} \leq(\mathrm{j}-1) \mathrm{a}_{\mathrm{j}-1, \mathrm{j}}=\mathrm{m}+1, \ldots \mathrm{p}$

then for a real $\alpha=-a_{n-1} / n a_{n}=\ldots .=-(n-m-1) a_{p+1} /(p+2) a_{p+2}$, where $\mathrm{p}=\mathrm{m}+1, \ldots . \mathrm{n}$ the polar derivative of $\mathrm{P}(\mathrm{z})$ with respect to $\alpha$ has $p$ zeros and they lie in

$|z| \leq\left|(n-p) a_{p}+\alpha(p+1) a_{p+1}\right|^{-1}\left\{-(n-p) a_{p}-\alpha(p+1) a_{p+1}+k(n-m) a_{m}+\right.$

$\alpha(\mathrm{m}+1) \mathrm{a}_{\mathrm{m}+1}+(\mathrm{n}-2 \mathrm{~m})(\mathrm{k}-1)\left|\mathrm{a}_{\mathrm{m}}\right|+$

$\left|(n-m) a_{m}+\alpha(m+1) a_{m+1}-(n-m+1) a_{m-1}-\alpha m a_{m}\right|$

$\left.\left.+(\mathrm{n}-\mathrm{m}+1) \mathrm{a}_{\mathrm{m}-1}+\mathrm{k}_{\mathrm{m}} \mathrm{ma} \mathrm{m}_{\mathrm{m}}-\mathrm{na}_{0}-\alpha \mathrm{a}_{1}+\left|\mathrm{na}_{0}+\alpha \mathrm{a}_{1}\right|\right)\right\}$

Proof: Let $\mathrm{P}(\mathrm{z})=\mathrm{a}_{0}+\mathrm{a}_{1} \mathrm{z}+\mathrm{a}_{2} \mathrm{z}^{2}+\ldots \ldots+\mathrm{a}_{\mathrm{n}} \mathrm{z}^{\mathrm{n}}$ be $\mathrm{a}$ polynomial of degree $n$.

Then the polar derivative of $\mathrm{P}(\mathrm{z})$ is given by $\mathrm{D}_{\alpha} \mathrm{P}(\mathrm{z})=\mathrm{n} \mathrm{P}(\mathrm{z})$

$+(\alpha-z) P^{\prime}(z)$. Then

$\mathrm{D}_{\alpha} \mathrm{P}(\mathrm{z})==\left[\mathrm{na}_{0}+\alpha \mathrm{a}_{1}\right]+\left[(\mathrm{n}-1) \mathrm{a}_{1}+2 \alpha \mathrm{a}_{2}\right] \mathrm{z}+\left[(\mathrm{n}-2) \mathrm{a}_{2}+\right.$ $\left.3 \alpha a_{3}\right] z^{2}+\ldots$

$+\left[(n-m+1) a_{m-1}+\alpha m a_{m}\right] z^{m-1}+\left[(n-m) a_{m}+\alpha(m+1) a_{m+1}\right] z^{m}+$ $\left[(\mathrm{n}-\mathrm{m}-1) \mathrm{a}_{\mathrm{m}+1}+\alpha(\mathrm{m}+2) \mathrm{a}_{\mathrm{m}+2}\right] \mathrm{z}^{\mathrm{m}+1}+$

$+\left[2 a_{n-2}+\alpha(n-1) a_{n-1}\right] z^{n-2}+\left[a_{n-1}+\alpha n a_{n}\right] z^{n-1}$.

As $\alpha=-a_{n-1} / n a_{n}=\ldots=-(n-m-1) a_{p+1} /(p+2) a_{p+2}$

$D_{\alpha} P(z)=\left[(n-p) a_{p}+\alpha(p+1) a_{p+1}\right] z^{p}+\left[(n-p+1) a_{p-1}+\alpha p a_{p}\right] z^{p-1}$ $+\left[(\mathrm{n}-\mathrm{p}+2) \mathrm{a}_{\mathrm{p}-2}+\alpha(\mathrm{p}-1) \mathrm{a}_{\mathrm{p}-1}\right]+\ldots \ldots$.

$+\left[(n-2) a_{2}+3 \alpha a_{3}\right] z^{2} .+\left[(n-1) a_{1}+2 \alpha a_{2}\right] z+\left[n a_{0}+\alpha a_{1}\right]$

Now consider the polynomial $\mathrm{Q}(\mathrm{z})=(1-\mathrm{z}) \mathrm{D}_{\alpha} \mathrm{P}(\mathrm{z})$ so that

$\mathrm{Q}(\mathrm{z})=-\left[(\mathrm{n}-\mathrm{p}) \mathrm{a}_{\mathrm{p}}+\alpha(\mathrm{p}+1) \mathrm{a}_{\mathrm{p}+1}\right] \mathrm{z}^{\mathrm{p}+1}+\left[(\mathrm{n}-\mathrm{p}) \mathrm{a}_{\mathrm{p}}+\alpha(\mathrm{p}+1) \mathrm{a}_{\mathrm{p}+1}-\right.$ $(n-p+1) a_{p-1}$

- $\left.\quad \alpha p a_{p} \quad\right] z^{p}+\ldots . .+\left[(n-m-1) a_{m+1}+\alpha(m+2) a_{m+2}-(n-m) a_{m}-\right.$ $\left.\alpha(\mathrm{m}+1) \mathrm{a}_{\mathrm{m}+1}\right] \mathrm{z}^{\mathrm{m}+1}$

$+\left[(n-m) a_{m}+\alpha(m+1) a_{m+1}-(n-m+1) a_{m-1}-\alpha m a_{m}\right] z^{m}+\left[(n-m+1) a_{m-}\right.$ 


\section{International Journal of Science and Research (IJSR) \\ ISSN (Online): 2319-7064}

Index Copernicus Value (2013): 6.14 | Impact Factor (2014): 5.611

$\left.+\alpha m a_{m}-(n-m+2) a_{m-2}-\alpha(m-1) a_{m-1}\right] z^{m-1}+\ldots$

$+\left[(n-2) a_{2}+3 \alpha a_{3}-(n-1) a_{1}-2 \alpha a_{2}\right] z^{2}+\left[(n-1) a_{1}+2 \alpha a_{2}-n a_{0}\right.$

$\left.-\alpha \mathrm{a}_{1}\right] \mathrm{z}$

$+\left[\mathrm{na}_{0}+\alpha \mathrm{a}_{1}\right]$.

Now if $|z|>1$ then $|z|^{-(n-i)}<1$ for $i=n-1, \ldots, n-p$

Further,

$|\mathrm{Q}(\mathrm{z})| \geq\left|(\mathrm{n}-\mathrm{p}) \mathrm{a}_{\mathrm{p}}+\alpha(\mathrm{p}+1) \mathrm{a}_{\mathrm{p}+1}\right||z|^{\mathrm{p}+1}-\left\{\mid(\mathrm{n}-\mathrm{p}) \mathrm{a}_{\mathrm{p}}+\alpha(\mathrm{p}+1) \mathrm{a}_{\mathrm{p}+1}\right.$

$-(n-p+1) a_{p-1}-\left.\alpha p a_{p}|| z\right|^{p}+\mid(n-p+1) a_{p-1}-\alpha p a_{p}-(n-p+2) a_{p-2}$

$-\left.\alpha(\mathrm{p}-1) \mathrm{a}_{\mathrm{p}-1}|| z\right|^{\mathrm{p}-1}+\ldots+\mid(\mathrm{n}-\mathrm{m}-1) \mathrm{a}_{\mathrm{m}+1}+\alpha(\mathrm{m}+2) \mathrm{a}_{\mathrm{m}+2}-(\mathrm{n}-\mathrm{m}) \mathrm{a}_{\mathrm{m}}-$ $\left.\alpha(\mathrm{m}+1) \mathrm{a}_{\mathrm{m}+1}|| \mathrm{z}\right|^{\mathrm{m}+1}$

$+\left|(n-m) a_{m}+\alpha(m+1) a_{m+1}-(n-m+1) a_{m-1}-\alpha m a_{m}\right||z|^{m}$

$+\left|(n-m+1) a_{m-1}+\alpha m a_{m}-(n-m+2) a_{m-2}-\alpha(m-1) a_{m-1}\right||z|^{m-1}+\ldots .$.

$+\left|(n-2) a_{2}+3 \alpha a_{3}-(n-1) a_{1}-2 \alpha a_{2}\right||z|^{2}+\mid(n-1) a_{1}+2 \alpha a_{2}-n a_{0}-$ $\alpha \mathrm{a}_{1}|| \mathrm{z} \mid$

$\left.+\left|\mathrm{na}_{0}+\alpha \mathrm{a}_{1}\right|\right\}$

$\geq\left|(n-p) a_{p}+\alpha(p+1) a_{p+1}\right||z|^{p}\left[|z|-\left|(n-p) a_{p}+\alpha(p+1) a_{p+1}\right|^{-1}\{\mid(n-\right.$ p) $\mathrm{a}_{\mathrm{p}}+\alpha(\mathrm{p}+1) \mathrm{a}_{\mathrm{p}+1}$

- $(n-p+1) a_{p-1}-\alpha p a_{p}|+|(n-p+1) a_{p-1}-\alpha p a_{p}-(n-p+2) a_{p-2}-\alpha(p-$ 1) $\left.\mathrm{a}_{\mathrm{p}-1}|| \mathrm{z}\right|^{-1}+\ldots \ldots$

$+\left|(n-m-1) a_{m+1}+\alpha(m+2) a_{m+2}-(n-m) a_{m}-\alpha(m+1) a_{m+1}\right||z|^{-(p-m-1)}$

$+\left|(n-m) a_{m}+\alpha(m+1) a_{m+1}-(n-m+1) a_{m-1}-\alpha m a_{m} \| z\right|^{-(p-m)}$

$+\left|(n-m+1) a_{m-1}+\alpha m a_{m}-(n-m+2) a_{m-2}-\alpha(m-1) a_{m-1}\right||z|^{-(p-m+1)}+\ldots$.

$+\left|(n-2) a_{2}+3 \alpha a_{3}-(n-1) a_{1}-2 \alpha a_{2}\right||z|^{-(p-2)}$

$+\left|(n-1) a_{1}+2 \alpha a_{2}-n a_{0}-\alpha a_{1}\left\|\left.z\right|^{-(p-1)}+\left|n a_{0}+\alpha a_{1} \| z\right|^{-p}\right\}\right]$

$\geq\left|(n-p) a_{p}+\alpha(p+1) a_{p+1}\right||z|^{p}\left[|z|-\left|(n-p) a_{p}+\alpha(p+1) a_{p+1}\right|^{-1}\{\mid(n-\right.$ p) $\mathrm{a}_{\mathrm{p}}+\alpha(\mathrm{p}+1) \mathrm{a}_{\mathrm{p}+1}$

- $(n-p+1) a_{p-1}-\alpha p a_{p}|+|(n-p+1) a_{p-1}-\alpha p a_{p}-(n-p+2) a_{p-2}-\alpha(p-$

1) $\mathrm{a}_{\mathrm{p}-1} \mid+\ldots \ldots$

$+\left|(n-m-1) a_{m+1}+\alpha(m+2) a_{m+2}-(n-m) a_{m}-\alpha(m+1) a_{m+1}\right|$

$+\left|(n-m) a_{m}+\alpha(m+1) a_{m+1}-(n-m+1) a_{m-1}-\alpha m a_{m}\right|$

$+\left|(n-m+1) a_{m-1}+\alpha m a_{m}-(n-m+2) a_{m-2}-\alpha(m-1) a_{m-1}\right|+\ldots .$.

$+\left|(n-2) a_{2}+3 \alpha a_{3}-(n-1) a_{1}-2 \alpha a_{2}\right|+\mid(n-1) a_{1}+2 \alpha a_{2}-n a_{0}-\alpha a_{1}$

$|+| \mathrm{na}_{0}+\alpha \mathrm{a}_{1} \mid$

$\geq\left|(n-p) a_{p}+\alpha(p+1) a_{p+1}\right||z|^{p}\left[|z|-\left|(n-p) a_{p}+\alpha(p+1) a_{p+1}\right|^{-1}\left\{\mid(n-p) a_{p}\right.\right.$

$+\alpha(\mathrm{p}+1) \mathrm{a}_{\mathrm{p}+1}$

- $(n-p+1) a_{p-1}-\alpha p a_{p}|+|(n-p+1) a_{p-1}-\alpha p a_{p}-(n-p+2) a_{p-2}-\alpha(p-$

1) $\mathrm{a}_{\mathrm{p}-1} \mid+\ldots \ldots$

$+\mid(n-m-1) a_{m+1}+\alpha(m+2) a_{m+2}-k(n-m) a_{m}-\alpha(m+1) a_{m+1}+k(n-$

$\mathrm{m}) \mathrm{a}_{\mathrm{m}}-(\mathrm{n}-\mathrm{m}) \mathrm{a}_{\mathrm{m}}$

$+\left|(n-m) a_{m}+\alpha(m+1) a_{m+1}-(n-m+1) a_{m-1}-\alpha m a_{m}\right|$

$+\mid(n-m+1) a_{m-1}+\alpha k m a_{m}-(n-m+2) a_{m-2}-\alpha(m-1) a_{m-1}-$

$\alpha \mathrm{kma}_{\mathrm{m}}+\alpha \mathrm{ma}_{\mathrm{m}} \mid+\ldots$

$+\left|(n-2) a_{2}+3 \alpha a_{3}-(n-1) a_{1}-2 \alpha a_{2}\right|+\mid(n-1) a_{1}+2 \alpha a_{2}-n a_{0}-$ $\left.\left.\alpha \mathrm{a}_{1}|+| \mathrm{na}_{0}+\alpha \mathrm{a}_{1} \mid\right\}\right]$

$\geq\left|(n-p) a_{p}+\alpha(p+1) a_{p+1}\right||z|^{p}\left[|z|-\left|(n-p) a_{p}+\alpha(p+1) a_{p+1}\right|^{-1}\{-(n-\right.$ p) $a_{\mathrm{p}}-\alpha(\mathrm{p}+1) \mathrm{a}_{\mathrm{p}+1}$

$+(n-p+1) a_{p-1}+\alpha p a_{p}-(n-p+1) a_{p-1}-\alpha p a_{p}+(n-p+2) a_{p-2}+\alpha(p-$

1) $\mathrm{a}_{\mathrm{p}-1} \mid+\ldots \ldots$

-(n-m-1) $a_{m+1}-\alpha(m+2) a_{m+2}+k(n-m) a_{m}+\alpha(m+1) a_{m+1}+(k-1)(n-$

$\mathrm{m})\left|\mathrm{a}_{\mathrm{m}}\right|$

$+\left|(n-m) a_{m}+\alpha(m+1) a_{m+1}-(n-m+1) a_{m-1}-\alpha m a_{m}\right|-(n-m+2) a_{m-2}-$

$\alpha(\mathrm{m}-1) \mathrm{a}_{\mathrm{m}-1}$

$+(\mathrm{n}-\mathrm{m}+1) \mathrm{a}_{\mathrm{m}-1}+\alpha \mathrm{kma}_{\mathrm{m}}+(1-\mathrm{k}) \mathrm{m}\left|\mathrm{a}_{\mathrm{m}}\right|+\ldots-(\mathrm{n}-1) \mathrm{a}_{1}-2 \alpha \mathrm{a}_{2}+(\mathrm{n}-$

2) $\mathrm{a}_{2}+3 \alpha \mathrm{a}_{3}$

$\left.\left.-\mathrm{na}_{0}-\alpha \mathrm{a}_{1}+(\mathrm{n}-1) \mathrm{a}_{1}+2 \alpha \mathrm{a}_{2}+\left|\mathrm{na}_{0}+\alpha \mathrm{a}_{1}\right|\right\}\right)$

$\geq\left|(n-p) a_{p}+\alpha(p+1) a_{p+1}\right||z|^{p}\left[|z|-\left|(n-p) a_{p}+\alpha(p+1) a_{p+1}\right|^{-1}\{-(n-\right.$

p) $a_{p}-\alpha(p+1) a_{p+1}$

$+\mathrm{k}(\mathrm{n}-\mathrm{m}) \mathrm{a}_{\mathrm{m}}+\alpha(\mathrm{m}+1) \mathrm{a}_{\mathrm{m}+1}+(\mathrm{k}-1)(\mathrm{n}-\mathrm{m})\left|\mathrm{a}_{\mathrm{m}}\right|+\mid(\mathrm{n}-$

m) $a_{m}+\alpha(m+1) a_{m+1}-(n-m+1) a_{m-1}-\alpha m a_{m} \mid$

$\left.\left.+(\mathrm{n}-\mathrm{m}+1) \mathrm{a}_{\mathrm{m}-1}+\alpha \mathrm{kma}_{\mathrm{m}}+(1-\mathrm{k}) \mathrm{m}\left|\mathrm{a}_{\mathrm{m}}\right|-\mathrm{na}_{0}-\alpha \mathrm{a}_{1}+\left|\mathrm{na}_{0}+\alpha \mathrm{a}_{1}\right|\right\}\right]$

$\geq\left|(n-p) a_{p}+\alpha(p+1) a_{p+1}\right||z|^{p}\left[|z|-\left|(n-p) a_{p}+\alpha(p+1) a_{p+1}\right|^{-1}\{-(n-\right.$

p) $a_{p}-\alpha(p+1) a_{p+1}$

$+[\mathrm{k}(\mathrm{n}-\mathrm{m})+\mathrm{k} \alpha \mathrm{m}] \mathrm{a}_{\mathrm{m}}+\alpha(\mathrm{m}+1) \mathrm{a}_{\mathrm{m}+1}+(\mathrm{k}-1)(\mathrm{n}-2 \mathrm{~m})\left|\mathrm{a}_{\mathrm{m}}\right|$ $+\left|(n-m) a_{m}+\alpha(m+1) a_{m+1}-(n-m+1) a_{m-1}-\alpha m a_{m}\right|$

$+($ n.m +1$\left.\left.) a_{m-1}-n a_{0}-\alpha a_{1}+\left|n a_{0}+\alpha a_{1}\right|\right\}\right]$

$>0$ if $|z|>\left|(n-p) a_{p}+\alpha(p+1) a_{p+1}\right|^{-1}\left\{-(n-p) a_{p}-\alpha(p+1) a_{p+1}\right.$

$+[\mathrm{k}(\mathrm{n}-\mathrm{m})+\mathrm{k} \alpha \mathrm{m}] \mathrm{a}_{\mathrm{m}}+\alpha(\mathrm{m}+1) \mathrm{a}_{\mathrm{m}+1}+(\mathrm{k}-1)(\mathrm{n}-2 \mathrm{~m})\left|\mathrm{a}_{\mathrm{m}}\right|$

$+\left|(n-m) a_{m}+\alpha(m+1) a_{m+1}-(n-m+1) a_{m-1}-\alpha m a_{m}\right|$

$\left.\left.+(n . m+1) a_{m-1}-n a_{0}-\alpha a_{1}+\left|n a_{0}+\alpha a_{1}\right|\right\}\right]$

This shows that if $|z||>|(n-p) a_{p}+\left.\alpha(p+1) a_{p+1}\right|^{-1}\left\{-(n-p) a_{p}-\right.$ $\alpha(\mathrm{p}+1) \mathrm{a}_{\mathrm{p}+1}$

$+[\mathrm{k}(\mathrm{n}-\mathrm{m})+\mathrm{k} \alpha \mathrm{m}] \mathrm{a}_{\mathrm{m}}+\alpha(\mathrm{m}+1) \mathrm{a}_{\mathrm{m}+1}+(\mathrm{k}-1)(\mathrm{n}-2 \mathrm{~m})\left|\mathrm{a}_{\mathrm{m}}\right|$

$+\left|(n-m) a_{m}+\alpha(m+1) a_{m+1}-(n-m+1) a_{m-1}-\alpha m a_{m}\right|$

$\left.\left.+(\mathrm{n} . \mathrm{m}+1) \mathrm{a}_{\mathrm{m}-1}-\mathrm{na}_{0}-\alpha \mathrm{a}_{1}+\left|\mathrm{na}_{0}+\alpha \mathrm{a}_{1}\right|\right\}\right]$, then $\mathrm{Q}(\mathrm{z})>0$.

Hence all the zeros of $Q(z)$ with $|z|>1$ lie in $|z| \leq\left|(n-p) a_{p}+\alpha(p+1) a_{p+1}\right|^{-1}\left\{-(n-p) a_{p}-\alpha(p+1) a_{p+1}\right.$ $+[\mathrm{k}(\mathrm{n}-\mathrm{m})+\mathrm{k} \alpha \mathrm{m}] \mathrm{a}_{\mathrm{m}}+\alpha(\mathrm{m}+1) \mathrm{a}_{\mathrm{m}+1}+(\mathrm{k}-1)(\mathrm{n}-2 \mathrm{~m})\left|\mathrm{a}_{\mathrm{m}}\right|$ $+\left|(n-m) a_{m}+\alpha(m+1) a_{m+1}-(n-m+1) a_{m-1}-\alpha m a_{m}\right|$

$\left.\left.+(n-m+1) a_{m-1}-n a_{0}-\alpha a_{1}+\left|n a_{0}+\alpha a_{1}\right|\right\}\right]$.

But those zeros of $\mathrm{Q}(\mathrm{z})$ whose modulus is less than or equal to 1 ,already satisfy the above inequality, since all the zeros of $\mathrm{D}_{\alpha} \mathrm{P}(\mathrm{z})$ are also the zeros of $\mathrm{Q}(\mathrm{z})$ as they lie in the circle defined by the above inequality.Hence proof of the theorem is complete.

Corollary : 9 Let $\mathrm{P}(\mathrm{z})=\sum_{i=0}^{n} a_{\mathrm{i}} \mathrm{z}^{\mathrm{i}}$ be a polynomial of degree $\mathrm{n}$ such that

$a_{0} \leq a_{1} \leq \ldots \leq a_{m} \geq a_{m+1} \geq \ldots a_{p-1} \geq a_{p}$

$(n-i) a_{i} \leq a_{i+1}, i=0,1,2, \ldots . m-1$

$\mathrm{ja}_{\mathrm{j}} \leq(\mathrm{j}-1) \mathrm{a}_{\mathrm{j}-1, \mathrm{j}}=\mathrm{m}+1, \ldots \mathrm{p}$

then for a real $\alpha=-a_{n-1} / n a_{n}=\ldots .=-(n-m-1) a_{p+1} /(p+2) a_{p+2}$, where $p=m+1, \ldots . n$ the polar derivative of $P(z)$ with respect to $\alpha$ has $\mathrm{p}$ zeros and they lie in

$|z| \leq\left|(n-p) a_{p}+\alpha(p+1) a_{p+1}\right|^{-1}\left\{-(n-p) a_{p}-\alpha(p+1) a_{p+1}+(n-m) a_{m}+\right.$ $\alpha(\mathrm{m}+1) \mathrm{a}_{\mathrm{m}+1}$

$+\left|(n-m) a_{m}+\alpha(m+1) a_{m+1}-(n-m+1) a_{m-1}-\alpha m a_{m}\right|$

$\left.\left.+(n-m+1) a_{m-1}+\alpha m a_{m}-n a_{0}-\alpha a_{1}+\left|n a_{0}+\alpha a_{1}\right|\right)\right\}$

The above corollary is obtained by taking $\mathrm{k}=1$ in theorem 8 .

\section{References}

[1] A.Aziz and B. A. Zargar, Some extensions of Eneström -Kakeya Theorem, Glasnik Matematiki, 31,(1996), 239244 ..

[2] G. Eneström, Remarquee sur un théorém relatif aux racines de l'equation $\mathrm{a}_{\mathrm{n}}+\ldots .+\mathrm{a}_{0}=0$ oü tous les coefficient sont et positifs, Tôhoku Math.J 18(1920), 34-36.

[3] A.Joyal, G.Labelle and Q.I.Rahman, On the location of zeros of polynomial, Canad. Math. Bull. 10,(1967),5363.

[4] S.KAKEYA, on the limits of the roots of an algebraic equation with positive coefficient, Tôhoku Math.J 2(1912-1913),140-142.

\section{Author Profile}

K. Sravani, School of Mathematics and Statistics, University of Hyderabad - 500046, India

K. Anoosha School of Mathematics and Statistics, University of Hyderabad - 500046, India

G.L. Reddy School of Mathematics and Statistics, University of Hyderabad - 500046, India

\section{Volume 4 Issue 11, November 2015}

